\title{
LA ALFABETIZACIÓN ACADÉMICA DESDE UNA PERSPECTIVA MULTIDIMENSIONAL
}

\section{ACADEMIC LITERACY FROM A MULTIDIMENSIONAL PERSPECTIVE}

Ana Pujol Dahme

Universidad de Barcelona

\section{Resumen}

A partir de la educación secundaria los estudiantes acceden a las diferentes culturas escritas de las disciplinas especializadas. La cultura escrita está inexorablemente vinculada a la práctica de cualquier disciplina académica. Por eso, para integrarse a una comunidad disciplinar es necesario dominar la escritura académica. Esto requiere un nivel avanzado de alfabetización académica. La alfabetización avanzada consiste en desarrollar el dominio de nuevos géneros y las formas que cada comunidad disciplinar utiliza para crear y compartir el conocimiento. En este artículo se propone una perspectiva multidimensional para ilustrar el reto que supone para los estudiantes llegar a desarrollar esta alfabetización en los trabajos de investigación. Las dimensiones analizadas son la lingüística, la discursiva y la semiótica visual. De la dimensión lingüística se destacan las características léxico-gramaticales como la diversidad léxica, la complejidad sintáctica y la densidad léxica. De la dimensión discursiva se revisa la argumentación. La dimensión semiótica visual se explica desde las macrofunciones de la Lingüística Sistémico Funcional. Se utilizan datos del corpus TARBUC (Treballs Acadèmics de Recerca de Batxillerat i Universitat en Català), el primer corpus en lengua catalana de trabajos de investigación de bachillerato y de máster universitario, en las disciplinas de biología e historia, para aportar ejemplos de cada una de las dimensiones. Finalmente se presentan algunas conclusiones e implicaciones didácticas.

Palabras clave: Escritura académica, lingüística, argumentación, semiótica visual

\section{Abstact}

From secondary school onwards, students have access to different written cultures of several specialized disciplines. Every academic discipline practice is linked to a specific written culture. In order to join a research community of practice, students' academic writing mastering is paramount, and consequently 
an advanced academic literacy level. Advanced literacy involves the command of new genres and forms used by each disciplinary community for knowledge creation and sharing. This paper proposes a multidimensional perspective with the aim of illustrating the challenges students have to overcome when acquiring academic literacy. Linguistic, discursive and visual semiotic dimensions are analysed. Within the linguistic dimension lexicogrammatical features are highlighted, such as lexical diversity, syntactic complexity, and lexical density. From the discursive dimension, argumentation is reviewed. The visual semiotic dimension is analysed using the macrofunctions from the Systemic Functional Linguistics as basis. Examples of each dimension are provided using data from the TARBUC corpus (Treballs Academics de Recerca en Batxillerat $\mathrm{i}$ Universitat en Català), the first Catalan-language research projects corpus including high school and Master in the disciplines of Biology and History. Finally, some conclusions and educational implications are presented.

Key-words: Academic writing, language, argumentation, visual semiotics.

\section{INTRODUCCIÓN}

La alfabetización es un sistema semiótico social (Halliday, 1978; Lemke, 1990), es una forma de acción social para crear significado con un sentido para la comunidad. Las personas utilizamos los recursos de una variedad de sistemas semióticos, es decir signos, para crear significado. Elegimos un modo u otro, según los objetivos que queremos conseguir en los contextos interpersonales o institucionales en el que nos encontramos. Por ejemplo, escribimos el signo lingüístico (en nuestro caso, las palabras, el texto) con el objetivo que sea relevante para, y reconocible por, los miembros de alguna comunidad (Lemke, 2002). La alfabetización es un proceso que se desarrolla en la sociedad y en el individuo. En el contexto social, esta evolución es especialmente dinámica, ya que sobre todo, los cambios tecnológicos, se suceden rápidamente y requieren de nuevas formas de alfabetización para poder adaptarse a estos cambios. En el ámbito académico, se crea y se comparte nuevo conocimiento a través de significados complejos, con diferentes sistemas de notación, como el escrito, el numérico y el visual, entre otros (Schleppegrell \& Colombi, 2002). La alfabetización académica, por tanto, debería consistir en fomentar que los estudiantes participen de las prácticas discursivas de las disciplinas para poder apropiarse del conocimiento (Carlino, 2013). Es decir, favorecer que escriban y lean como lo hacen los especialistas, los miembros de cada comunidad disciplinar. Cada comunidad tiene sus propias prácticas discursivas, es decir, sus miembros utilizan el lenguaje escrito con ciertos propósitos y de acuerdo con valores epistémicos propios (Bazerman, 2005; Carlino, 2005; Ivanic, 1998; Prior, 2006). 
La alfabetización es una parte fundamental de todas las disciplinas académicas, también de las científicas. Podríamos pensar que lo que constituye la ciencia es su contenido y que el conocimiento letrado sólo es una herramienta auxiliar para almacenar o transmitir conocimientos. Sin embargo, la evolución de la ciencia está relacionada con la evolución de los recursos gramaticales, que a su vez construyen el pensamiento científico (Halliday, 1993). El conocimiento letrado es intrínseco a la ciencia, como lo es la medición u observación (Norris \& Phillips, 2009). Por lo tanto, desarrollar el conocimiento letrado es importante para lograr el éxito académico y el desarrollo profesional (Tolchinsky \& Simó, 2001; Snow \& Biancarosa, 2003).

En el contexto académico, los estudiantes aprenden en diferentes asignaturas modos particulares de crear e interpretar significado; de argumentar y criticar; de integrar diferentes significados impresos, visuales, electrónicos; es decir, las formas que cada comunidad disciplinar utiliza para crear y compartir el conocimiento. Esto implica que los estudiantes han de desarrollar nuevas habilidades.

Todas estas características tienen implicaciones directas en el campo de la investigación y de la enseñanza. Por un lado, el tema de la alfabetización sigue suscitando interés como objeto de investigación, ya que por su propiedad dinámica se abren constantemente nuevas perspectivas de análisis. Por otro, el desarrollar cierta competencia en utilizar el lenguaje complejo, así como los diferentes modos semióticos supone una gran demanda para el alumno. Asimismo, implica un reto para el profesorado que todos los alumnos lleguen a adquirir una alfabetización avanzada. Por consiguiente, se necesita una descripción lingüística comprensiva de los registros que se dan en el entorno académico, para poder dotar a los estudiantes de las habilidades y conocimientos necesarios para hacer frente a la compleja tarea de utilizar el lenguaje para nuevos propósitos y en diferentes contextos (Biber, 2006).

Por otra parte, la escritura es un modo semiótico con una representación externa, altamente convencionalizada. Esta convención permite a los usuarios reconocerla, pero el aprendizaje de un sistema de notación, tal como la escritura, no consiste solamente en cambiar el modo semiótico. Hay un cambio cognitivo en el dominio y en la representación involucrado en dicho sistema de notación. Así que la escritura es útil no sólo para comunicar significado, sino que también es una herramienta epistémica ya que en ella se plantean unos problemas que hay que resolver. Esta actividad de resolución de problemas tiene lugar en dos espacios que interactúan constantemente, el espacio del problema del contenido (qué) y el espacio del problema retórico (cómo), donde el escritor tiene presente al lector e intenta resolver cómo transmitir de forma adecuada el contenido (Bereiter \& Scardamalia, 1987). 
Además, la escritura es un medio para participar en la sociedad. Desde la escuela primaria hasta más allá del nivel universitario, el lenguaje escrito permite integrar a sus miembros en las diferentes comunidades discursivas (Tolchinsky \& Simó, 2001).

Pero ¿̇cuáles son las características del lenguaje escrito especializado? En el presente artículo nos centraremos en la escritura académica del género de investigación, ya que, por ejemplo, en Cataluña los estudiantes de secundaria, 17-18 años de edad, deben realizar un trabajo de investigación como requisito académico de su plan de estudios. Este trabajo de investigación, que es el resultado de una investigación personal guiada por el profesor, debe ser presentado en forma escrita y permite que los estudiantes desarrollen la investigación en general, la discusión y las habilidades expresivas, de forma similar a como ocurre en las comunidades científicas. Por otra parte, en la universidad, los estudiantes deben presentar por escrito y defender oralmente, un trabajo de investigación cuando finalizan sus estudios de máster. Lo que significa que desde una etapa educativa temprana los estudiantes tienen que hacer frente no sólo con las habilidades de investigación, sino también con el lenguaje especializado de su disciplina. La adquisición por parte de los estudiantes del lenguaje especializado se refleja en sus producciones, es decir, sus textos.

Con este artículo se propone que una perspectiva multidimensional sobre la escritura académica permite dar cuenta de algunas de sus características $y$, por lo tanto, de los retos que han de superar los estudiantes a la hora de escribir trabajos de investigación.

Como veremos, la escritura académica es multidimensional y, por consiguiente, requiere de múltiples perspectivas para su análisis y comprensión. Las dimensiones que se abordan son: la lingüística, la discursiva y la semiótica visual. Para ilustrar con ejemplos algunas de las características se utiliza el Corpus TARBUC (Treballs Acadèmics de Recerca de Batxillerat i Universitat en Català), que está constituido por 114 trabajos de investigación en las disciplinas de biología e historia de los niveles de bachillerato y máster universitario. Para este artículo se tendrá en cuenta únicamente los datos relacionados con biología, ya que esta es representativa de las ciencias experimentales.

\section{LA AlFABEtizACIÓN ACADÉMICA AVANZADA: UNA PERSPECTIVA MULTIDIMENSIONAL}

La participación en el contexto académico, que se desarrolla a partir de la educación secundaria, requiere un conocimiento avanzado de alfabetización, ya que los estudiantes tienen que lidiar con disciplinas especializadas basadas en el conocimiento técnico (Halliday, 2004; Christie \& Derewianka, 2008; Schleppegrell \& Colombi, 2002). El término 
'alfabetización avanzada' refiere a la forma de crear significado a partir de la educación secundaria (Schleppegrell \& Colombi, 2002). Desde una perspectiva psicolingüística se considera la adolescencia, en este caso la edad comprendida entre los 15-18 años, como un punto de inflexión en el conocimiento y uso lingüístico, tanto a nivel léxico y sintáctico como a nivel de posicionamiento discursivo (Berman, 2007; Berman \& Verhoeven, 2002). La alfabetización avanzada consiste en realizar un progreso hacia el dominio de nuevos géneros (Uccelli, Dobbs, \& Scott, 2013). Por género se entiende un grupo de textos, que representa cómo los escritores usan el lenguaje para situaciones recurrentes. En un sentido amplio, el término género se puede caracterizar de la siguiente manera: cada género tiene un propósito específico, una estructura general, algunos rasgos lingüísticos específicos, y es compartida por los miembros de una comunidad (Hyland, 2004). Desde la Lingüística Sistémico Funcional, un género es una actividad orientada a un objetivo con un propósito comunicativo en la que los hablantes participan como miembros de nuestra cultura (Martin, 1984). En esta definición, género tiene un significado más amplio que cuando se refiere a tipos de texto; la cultura se define aquí como actividades generales. Por lo tanto, todas las actividades (por ejemplo, pedir una cita con el médico, escribir un ensayo) tienen su propio género. El trabajo de investigación, por su similitud al artículo de investigación, es considerado uno de los más avanzados géneros académicos (Schleppegrell, 2001), ya que es el resultado de un proceso complejo (Swales, 2004). Aunque los trabajos de investigación de los estudiantes y los artículos de investigación de los profesionales no son el mismo género, habitualmente el objetivo de la escritura académica es desarrollar la capacidad de escribir textos profesionales como, por ejemplo, el artículo de investigación (Conrad, 1996; Gardner \& Nesi, 2013; Russell \& Cortes, 2012). Cada género tiene su propio registro, es decir, unas características lingüísticas o repertorios lingüísticos propios. Sin embargo, algunas características lingüísticas son comunes a todas las disciplinas, es decir, son características para el género académico (Schleppegrell, 2001; Uccelli, Dobbs, \& Scott, 2013). Los estudiantes al escribir un trabajo de investigación han de dominar el registro académico común a todos los géneros académicos y, además, las características léxico-gramaticales propias del género de investigación.

Por otra parte, la investigación científica tiene dos pasos, uno es un proceso de construcción del conocimiento y el segundo es un modo discursivo, la argumentación, que está ligada a objetivos epistémicos valorados por cada disciplina (Sandoval \& Reiser, 2004). Argumentar con precisión es difícil; no obstante, es un requisito importante para la publicación de los artículos de investigación (Tolchinsky et al., 2013). Por este motivo, practicar la argumentación se ha convertido en una necesidad básica en las asignaturas de ciencias (Driver, Newton, \& Osborne, 2000). 
Otro aspecto a tener en cuenta es que los científicos se comunican, piensan y trabajan no sólo con palabras, sino también en espacios visuales definidos por representaciones gráficas. En los textos que producen suelen utilizar recursos verbales y no verbales, tales como imágenes, gráficos, tablas, que ayudan a entender mejor el contenido de los textos (Lemke, 1989). El trabajo de investigación que realiza todo el alumnado de bachillerato tiene por objetivo acercar a los estudiantes a la manera de comunicarse, pensar y trabajar de los científicos. Saber utilizar adecuadamente todos estos modos semióticos supone una gran demanda por parte del alumno. Por lo tanto, es fundamental que los estudiantes aprendan a utilizar los múltiples recursos no verbales, que amplían la comprensión de un tema.

Hasta aquí se han introducido los aspectos lingüísticos, argumentativos y semióticos visuales que se dan en los trabajos de investigación. A continuación se describen cada uno de ellos con más detalle.

\section{Dimensión lingüística}

Un registro es un conjunto de características léxicas y gramaticales que caracteriza a usos particulares del lenguaje (Bar-llan \& Berman, 2007; Biber, 1995; Halliday \& Hasan, 1989; Schleppegrell, 2001). Ya hemos visto que hay algunas características léxico-gramaticales que son comunes a todos los géneros académicos. Para describir el concepto teórico de léxico-gramática de la Lingüística Sistémico Funcional, sobre el cual está basado este artículo, hay que tener en cuenta algunas consideraciones previas. Podemos diferenciar dos enfoques respecto al lenguaje: el formal y el funcional. Respecto al primer enfoque, el lenguaje se entiende desde una perspectiva formal y sintáctica, sobre todo por la influencia de la obra de Chomsky. Esta aproximación al estudio del lenguaje asume que el lenguaje es esencialmente un fenómeno cognitivo, formado por un sistema complejo y abstracto de reglas. Así, las teorías formales conciben la gramática como regla y su objetivo es identificar las propiedades de esos sistemas de reglas. Desde esta perspectiva, la relación entre gramática y léxico es modular. La sintaxis, la morfología y el léxico son módulos independientes.

Por otro lado, las teorías funcionales entienden el lenguaje desde una perspectiva funcional y semántica. Conciben el lenguaje en términos de la función social a la que sirve. Se asume que el lenguaje es un recurso sistemático para expresar significado en un contexto. Así la gramática es un recurso para crear significado y el objetivo fundamental de la perspectiva funcional es describir los recursos disponibles en el lenguaje para expresar significado. Desde esta perspectiva la relación entre gramática y léxico es continua (Matthiessen, 2009). 
Tal como recogen Snow y Uccelli (2009) en una revisión muy comprensible y completa, las características léxico-gramaticales propias de la escritura académica son: una diversidad léxica alta, estructuras sintácticamente complejas y una gran densidad informativa con una alta proporción de palabras léxicas obtenidas generalmente a través de nominalizaciones. Estas características también son propias del lenguaje de la ciencia (Halliday, 2004; Halliday \& Martin, 1993). Hay un cierto paralelismo entre la evolución del lenguaje de la ciencia y el desarrollo del lenguaje desde la infancia (Christie \& Derewianka, 2008; Halliday, 2004; Veel, 1997). Por ejemplo, la densidad léxica es indicativa de los textos más abstractos, es decir, más alejados del 'aquí y ahora', una característica de los textos científicos. De manera similar, la densidad tiende a aumentar de la niñez a la edad adulta, especialmente en el discurso escrito (Halliday, 1993; Halliday, 2004; Veel, 1997).

La diversidad léxica. La escritura académica tiene generalmente una alta diversidad léxica, definida ésta como palabras diferentes utilizadas en un texto (Chafe \& Danielewicz, 1987). Se ha demostrado que la diversidad léxica se incrementa con un mayor conocimiento letrado (Malvern, Richards, Chipere, \& Durán, 2004). Desde una perspectiva de desarrollo son varios los estudios que han estudiado la diversidad léxica en diferentes lenguas (Berman \& Verhoeven, 2002; Johansson, 2008; Woerfel \& Yilmaz, 2011).

La complejidad sintáctica. La escritura académica se caracteriza por ser sintácticamente compleja, pero en el sentido de compresión sintáctica. Esta observación es importante, ya que cambia la idea estereotipada de que la complejidad en la escritura académica radica en el alto uso de oraciones subordinadas (Biber \& Gray, 2010). Estudios recientes de corpus sobre artículos de investigación profesionales han demostrado que la complejidad sintáctica se basa en modificadores incrustados en sintagmas nominales y no en la subordinación (Biber, Gray, \& Poonpon, 2011).

La densidad léxica, generalmente medida como la proporción de palabras léxicas o de contenido en relación con el número total de palabras. Las palabras léxicas transmiten la mayor parte del contenido semántico e información proposicional, por lo tanto, la alta densidad léxica se considera un buen indicador de la riqueza textual (Halliday, 1993 Malvern et al., 2004). No obstante, la densidad léxica contribuye a la complejidad del registro académico y, por lo tanto, hace que sea generalmente más 'duro' de leer. Así que los escritores deben mantener un equilibrio en el uso de la densidad léxica. La elección precisa y concisa de las palabras es más importante que una alta densidad léxica (Ventola, 1996). 
Los siguientes ejemplos de nuestro corpus permiten ilustrar la complejidad sintáctica y también la densidad léxica. La longitud de la oración se toma habitualmente como un índice de complejidad sintáctica (Uccelli, Dobbs, \& Scott, 2013). En el ejemplo (a) la oración del trabajo de investigación de bachillerato tiene 19 palabras y el ejemplo (b) del trabajo de final de máster tiene 26 palabras. En el ejemplo de bachillerato (a) hay 2 Sustantivos (NN) 1 adjetivo (JJ) y 1 frase preposicional (EN) que funcionan como modificadores del sustantivo antes de la oración de relativo (RC). En el ejemplo de máster (b) hay 5 sustantivos (NN), 3 adjetivos (JJ) y 3 frases preposicionales (IN) que funcionan como modificadores del sustantivo antes de la oración de relativo (RC). Como puede verse, el ejemplo de máster (b) es lexicalmente más denso, tiene más palabras de contenido (sustantivos, adjetivos, verbos y adverbios) que la oración de bachillerato (a). Vale la pena mencionar, que aunque el Corpus TARBUC está escrito en catalán se han traducido estos ejemplos al castellano.

a) La diabetes es una enfermedad crónica en la [que el nivel de NN

$$
\text { NN }
$$$$
\text { JJ IN RC }
$$

glucosa de la sangre se encuentra alterado].

b) El asma es una enfermedad respiratoria crónica, de carácter NN NN JJ JJ IN NN alérgico en la mayoría de casos, JJ IN NN IN NN

[que tiene una alta prevalencia en nuestra sociedad Europea actual]. RC

Estos ejemplos ilustran el desarrollo en la escritura académica. Aunque el ejemplo de máster es más complejo y denso, no significa que se adecue a los requisitos de los artículos de investigación. En un estudio previo (Pujol Dahme \& Selfa, 2015) hemos comparado la diversidad léxica, complejidad sintáctica y la densidad léxica de trabajos de investigación de estudiantes con artículos de investigación y concluimos que el llegar a usar estas características de forma adecuada es un proceso largo. Diferentes estudios (Baratta, 2010; Colombi, 2002) sugieren que a medida que se desarrollan las habilidades de escritura de los estudiantes, su densidad léxica y la estructura nominal crecen, mientras la complejidad gramatical, entendida como subordinación, disminuye.

\section{Dimensión discursiva}

Hay un amplio reconocimiento de la importancia de escribir y argumentar como parte del proceso educativo formal, ya que desarrolla 
el pensamiento crítico, y también aglutina a las comunidades disciplinarias (Bazerman, 1988). La ciencia avanza a través de la argumentación (Caamaño, 2010). El discurso de la ciencia en un nivel macro es argumentativo, en el sentido que su estructura está formada para apoyar las conclusiones sobre la base de razones y evidencias (Norris \& Phillips, 2009). El proceso de investigación consta de una formulación de un problema o de una hipótesis, un diseño de la investigación, recopilación e interpretación de los datos, y de estos se derivan unas conclusiones (White \& Frederiksen, 1998). Todos estos pasos se plasman por escrito a través de actos de habla: presentar los datos, describir el método, justificar las interpretaciones (Norris \& Phillips, 2009). Una vez que el proceso de investigación ha terminado, las conclusiones del proceso deben ser comunicadas a la comunidad científica por medio de artículos, conferencias, etc. para su debate. Esta comunicación es básicamente argumentativa. Por lo tanto, la argumentación es el modo discursivo directamente vinculado al artículo de investigación (Sampson \& Clark, 2008; Sandoval \& Reiser, 2004). La argumentación, al igual que la narración y exposición, tiene una estructura específica que cumple una función concreta, en este caso, convencer a la comunidad científica. La estructura que ha tenido mayor influencia en estudios sobre la enseñanza de ciencias es la propuesta por Toulmin (1985). Este matemático y filósofo inglés detalló los elementos que deberían de aparecer en un argumento para que este resulte convincente. Estos son: aserciones, datos y garantías (Tolchinsky et al., 2013).

La aserción (claim) es una declaración hecha por el autor que requiere de la aceptación por parte del lector y que el autor deberá sustentar esta afirmación general a lo largo del trabajo. En un trabajo de máster del Corpus TARBUC podemos ver la siguiente aserción.

c) Según el estímulo que han recibido las células $T$ quedan reprogramadas y responden con diferentes perfiles de citosinas frente a un nuevo estímulo.

Esta aserción es bastante categórica, por lo que el autor deberá sustentarla con datos.

Los datos (data) son las evidencias que sustentan la aserción y es la base de la persuasión.

d) Diferentes estudios apoyan la hipótesis de que la generación de Tregs puede ser debida a un cambio de su programa de transcripción y que condiciona su respuesta a los nuevos estímulos.

Vemos que en este ejemplo la evidencia está formada por resultados empíricos de otras investigaciones, no obstante no cita los autores de los estudios lo que hace que pierda fuerza esta evidencia. 
La garantía (warrant) relaciona los datos a la aserción y muestra que la evidencia es relevante. La garantía permite a otro investigador replicar el estudio, es decir, obtener similares resultados y conclusiones parecidas.

e) e) Ratones SEB2: recibían dos dosis altas de SEB separadas por 72 horas. Este es un modelo de shock séptico causado por SAG SEB en ratones Balb / c.

En este ejemplo, extraído del apartado de método de un trabajo de investigación de máster, se especifica cómo se ha suministrado el tratamiento a los ratones, lo que funciona como garantía.

Estos serían los elementos básicos del modelo de Toulmin, no obstante hay otros elementos (ver Tolchinsky et al., 2013). Por otra parte, Rodríguez Bello (2004) explica con detalle este modelo y propone un esquema didáctico interesante para estudiantes que se han de enfrentar a cualquier texto argumentativo. Para concluir este apartado recordemos que los principiantes que se integran en una comunidad disciplinaria deben adquirir la capacidad de generar un argumento de manera convincente, es decir, deberían ser coherentes con los criterios utilizados por su comunidad disciplinar (Sampson \& Clark, 2008; Sandoval \& Reiser, 2004).

\section{Dimensión semiótica visual}

La semiótica social tiene como objetivo explicar cómo creamos significado con todos los recursos a nuestra disposición: lingüísticos, pictóricos, gestuales, musicales, etc. (Kress \& Van Leeuwen, 1996; Lemke, 1989). Si consideramos que toda comunicación humana es multimodal, ya que se interrelacionan diferentes modos, como por ejemplo, el textual, visual, auditivo para crear significado (Lemke, 1998), también hemos de considerar el 'texto' como multimodal, ya que en él se combinan signos lingüísticos y no lingüísticos como por ejemplo, dibujos, gráficos, colores, etc. (Kucer, 2005).

Los trabajos de investigación también reflejan esta multimodalidad, son hipertextos primitivos, o mejor dicho, híbridos semióticos (Lemke, 1998). Los científicos no producen solamente un texto verbal lineal cuando se comunican. Usan representaciones gráficas (figuras, mapas, tablas, gráficos y fotografías) y expresiones matemáticas, porque piensan en espacios imaginarios visuales. El uso de esos recursos multimodales les permite multiplicar y modular el significado (Lemke, 1998). En otras palabras, un concepto científico no incluye todas las representaciones posibles, de hecho ningún tipo de concepto lo incluye, pero en el caso del concepto científico, este se articula a través de diferentes modos de representación para conseguir un significado completo. Además, no hay 
una traducibilidad directa e inequívoca entre los diferentes modos, por lo que la combinación de los mismos modula el significado. El ser capaz de utilizar un concepto científico, y por lo tanto para entenderlo en la forma en que un científico lo hace, requiere saber coordinar e interpretar con soltura los aspectos verbales, visuales y matemáticos. Desde la Lingüística Sistémico Funcional en el nivel de contexto de la situación, es decir del registro, se distingue entre campo (field): lo que se lleva a cabo; modo (mode): el canal de la comunicación y tenor (tenor): las relaciones entre los participantes. Estos tres valores del registro tienen su realización lingüística en las macrofunciones (Halliday, 1978), esto es, lo que sucede en el nivel de contexto de la situación en términos de campo, tenor y modo corresponde a lo que sucede en el nivel lingüístico con las tres macro-funciones: ideacional, interpersonal y textual. El lenguaje sirve simultáneamente a las 3 macrofunciones que se proyectan en el texto (Halliday, 1978), la ideacional, la interpersonal y la textual. La función ideacional incluye recursos para la creación de contenido; la textual, recursos para la organización de textos; y la función interpersonal incluye recursos para interactuar. Estas macrofunciones se relacionan con el lenguaje oral o escrito, pero en los recursos no verbales se pueden hacer corresponder con la función presentacional, orientacional y organizacional (Lemke, 1998), ya que como hemos mencionado antes, la naturaleza misma del ámbito científico hace que las ideas no se codifican sólo con recursos verbales. Evidentemente, no todas las relaciones que se pueden realizar lingüísticamente se pueden traducir visualmente y vice-versa. La posibilidad de realización en un modo semiótico $u$ otro depende del modo mismo, de la cultura y está determinada histórica- y socialmente (Kress \& van Leeuwen, 1996).

A continuación explicaremos estas funciones con ejemplos concretos extraídos de los trabajos de investigación del corpus TARBUC.

Función ideacional o presentacional: Representa los objetos externos e internos a nosotros, y sus relaciones. En el modo semiótico de la escritura esta función se expresa a través de la transitividad, las relaciones, los participantes semánticos y las circunstancias (cuándo, dónde, el porqué, cómo, etc.). En la oración siguiente (f), extraída del corpus TARBUC, en concreto de trabajos de investigación en biología de bachillerato, vemos que se establece el tópico o tema la hormona ADH y la relación se establece por medio de verbos de acción: actúa, reteniendo, necesita.

f) (f) El ADH es una hormona que actúa a nivel renal reteniendo el agua cuando el cuerpo la necesita.

En cambio, en la comunicación visual esta relación se puede crear por medio de vectores (Kress \& van Leeuwen, 1996). En la imagen 1 se 
presenta la división celular necesaria para la reproducción sexual o meiosis.

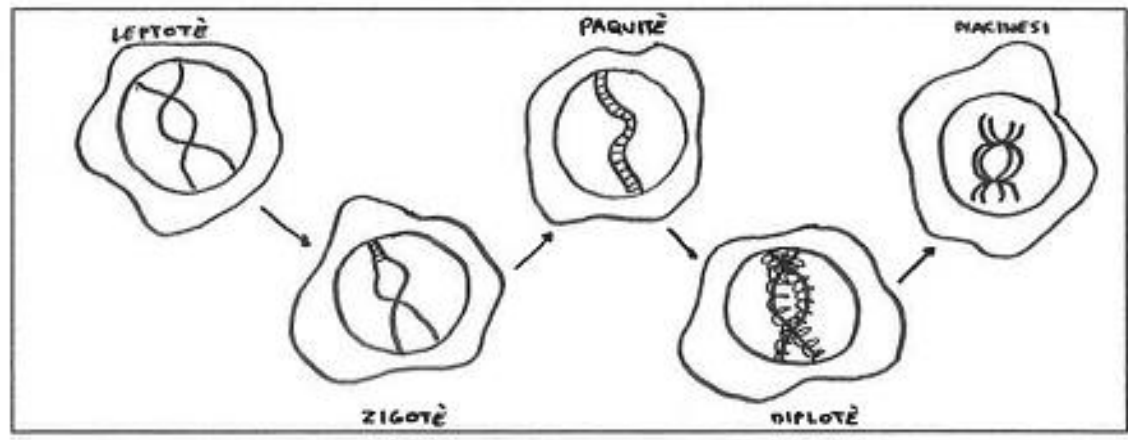

Esquema de la profase meiotica I

Imagen 1: Esquema de la división celular. (Corpus TARBUC)

Como vemos en la imagen 1, la acción se establece con vectores que relacionan las diferentes fases.

Hemos visto que la función ideacional o presentacional se puede expresar verbalmente o visualmente. Pero, lo que sería adecuado es expresar esta función de forma coordinada, es decir, los trabajos de investigación deberían incluir en el texto referencias al modo visual. Como ya hemos mencionado, es este ir y volver del texto a las imágenes, gráficos, etc. Io que proporciona un significado global claro y comprensible. Además, es la forma mediante la cual los científicos construyen y comunican la información.

Función interpersonal u orientacional: Cualquier modelo semiótico representa una relación social entre el emisor, el receptor y el objeto representado. En una interacción siempre hay alguno de los cuatro actos de habla basados "en ofrecimiento" y "demanda" (Halliday, 1985). En el caso de imágenes o diagramas se crea una relación hacia la escena (íntima, distante, superior) o se puede crear una orientación evaluativa hacia la escena (trágica, cómica, sorprendente) (Lemke, 1998). En ciencia, las imágenes que se utilizan son diagramas, gráficos y mapas y están codificados con una actitud objetiva, ofrecen información, normalmente por medio de la perspectiva y el ángulo. Así como para entender un texto escrito el lector debe saber decodificar el texto, para poder interpretar una imagen hay que conocer cómo se codifican en imágenes los significados interpersonales. La distancia es determinante en las relaciones sociales. Esto se entiende más fácilmente si analizamos imágenes fotográficas.

En la imagen 2 hay una distancia personal cercana, vemos la cabeza y los hombros de la persona, es decir, es un plano medio, además 
la relación entre el personaje y el espectador se crea por la mirada directa. El personaje 'pide' al observador que entre en una 'relación social' con él. En un texto escrito equivaldría a una apelación directa al lector.

En cambio, en la imagen 3 el personaje está distante, diluido en el entorno, plano panorámico. El hecho de que sea lejano hace que se destaque la soledad del personaje ante la dureza del invierno. No hay conexión visual, por tanto el espectador toma el rol 'de observador invisible'. Lingüísticamente no hay inclusión del lector. En este caso, hay un 'ofrecimiento' por parte del personaje como objeto de contemplación, de información impersonal.

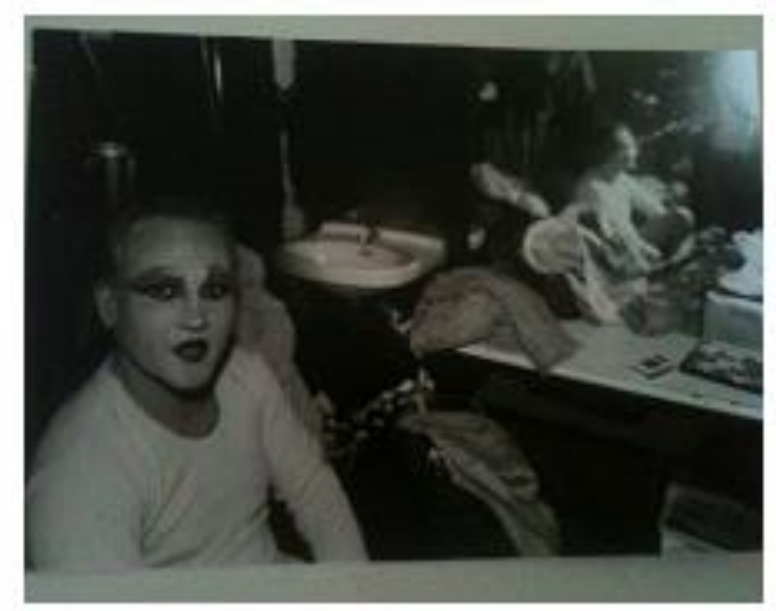

Imagen 2: Café-Teatro Llantiol. Fuente propia.

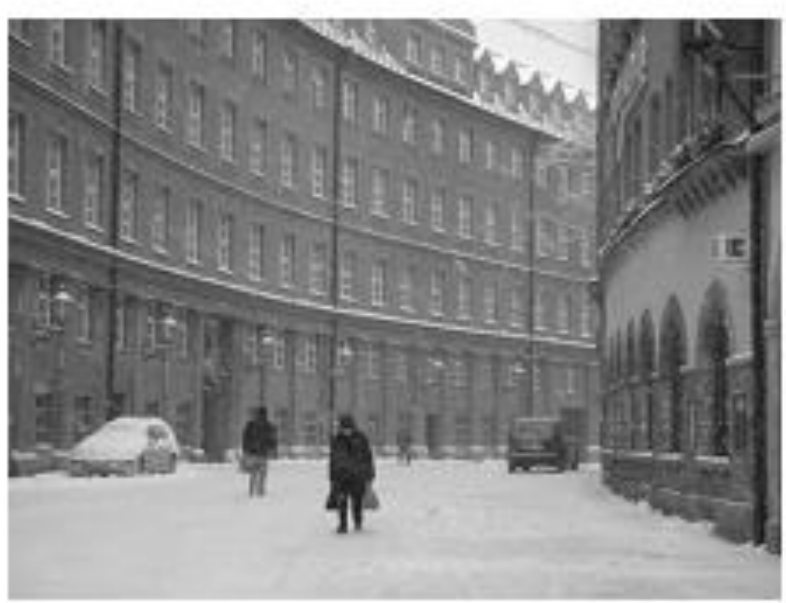

Imagen 3: Munich 2005. Fuente propia.

Sin embargo, en ciencia donde se utilizan gráficos y tablas, normalmente se codifica buscando una actitud objetiva. Esto se 
consigue con un ángulo frontal, se neutralizan así las posibles distorsiones que provoca la perspectiva. Pensemos, por ejemplo, en un cubo dibujado con perspectiva, los lados no parecerán igual de largos, como son en la realidad. Pero si nos situamos directamente frente al cubo, veremos sólo un cuadrado plano. Sin embargo, el uso de los ángulos, frontal y otros, no son del todo objetivos. Vemos en la imagen 4 un cubo plano.

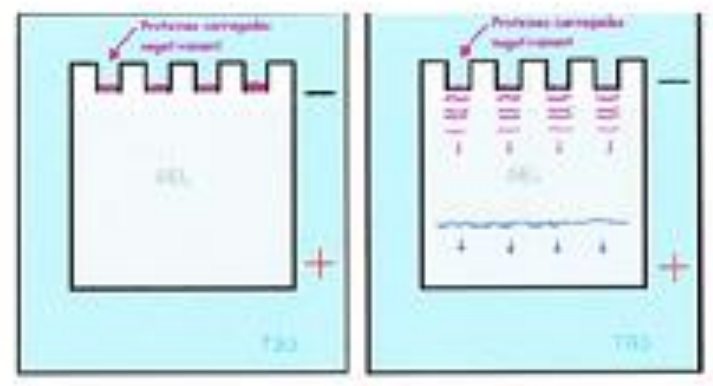

\begin{abstract}
En engegar el corrent, les proteines, carregases negativament carrea cap al pol positiu. Com que ne es poden weure les proteines s'afegeir el blau de bromotimol. Aquestes són les úniques particules que veiem i tenen el pes molecular mbs bax de manera que corren per dawant de les proteines. Quan les particules surten del gel es para Felectroforesi.
\end{abstract}

Imagen 4: Ángulo frontal en un trabajo de investigación de biologia. (Corpus TARBUC)

El ángulo frontal, imagen 4, es el de máxima implicación. Lingüísticamente tendría su equivalente en: "Así son las cosas, es como funciona" (Kress \& van Leeuwen, 1996).

Función textual u organizacional: así como cualquier texto escrito debe tener una coherencia, una cohesión interna y una adecuación al contexto, otros modos semióticos, como el modo visual, cumple esta función a través de la composición, principalmente. Aunque las imágenes pueden tener una coherencia y cohesión interna, en los textos científicos, donde se utilizan al mismo tiempo recursos visuales y verbales, estos últimos deberían hacer referencia a los recursos visuales. Ya que en este género textual las partes interactúan entre ellas y así pueden amplificar el significado del conjunto. El texto científico ni es lineal ni tampoco se lee de forma lineal, más bien constituye, como ya se ha mencionado, una forma primitiva de hipertexto (Lemke, 1998).

En este apartado se destaca la progresión temática, esto es, la organización del desarrollo de la información en un texto para dar cohesión. Se presenta un tema o tópico y se va añadiendo información nueva, rema. En el ejemplo (g) del Corpus TARBUC vemos cómo se organiza esta información. 
g) Este anticuerpo secundario [tema], tiene una enzima, la peroxidasa (HRP), que cataliza una reacción, provocada por los sustratos $A+B$, el producto de la cual da luz [rema]. Es precisamente esta luz[tema] la que detectarán las láminas intensificadores de la luz en el casete y gracias a ello podremos obtener el revelado[rema].

En el modo visual se presenta normalmente la información conocida, tema, a la izquierda y la nueva, rema, a la derecha de la imagen. En la imagen 5, trabajo de investigación biología, podemos ver el equivalente visual del ejemplo lingüístico (g).

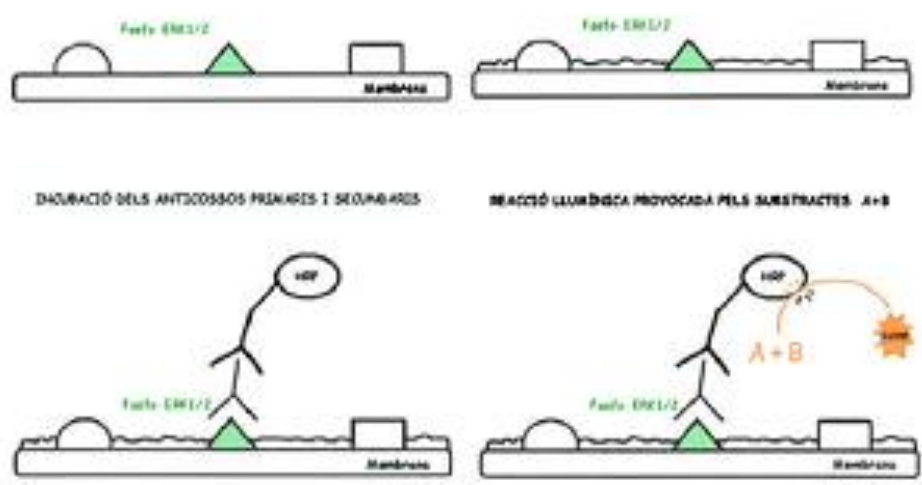

Imagen 5: Organización de tema y rema en un trabajo de investigación de biología. (Corpus TARBUC)

En esta imagen 5, vemos a la izquierda la información conocida, tema, se muestran las proteínas transferidas a la membrana. A la derecha, información nueva, rema, el bloqueo hecho para que ningún anticuerpo ocupe el espacio. Y en la imagen inferior a la derecha, queda reflejado la incubación con anticuerpos secundarios, éste tiene una enzima que provocará luz. Esta organización, tema-rema, da coherencia a la información.

Hasta aquí hemos visto algunos ejemplos de la semiótica visual. Evidentemente solo son pinceladas, ya que el tema es mucho más extenso.

A modo de resumen, hemos visto que el discurso verbal en el aula no es del todo comprensible sin activar otros sistemas semióticos, como el visual o el gestual. De hecho, no es posible activar sólo un sistema semiótico concreto. Movilizamos siempre más de un sistema, por consiguiente, siempre hay más de un posible significado en lo que expresamos. Los alumnos deberían desarrollar una alfabetización multimodal, que se puede definir como la capacidad para recibir y analizar la información recibida por diferentes modos para transformarlas en nuevo conocimiento. Por lo tanto, en la educación del s. XXI, 
caracterizada por las nuevas tecnologías, en que las herramientas digitales se imponen con fuerza en las aulas y fuera de ellas, el hecho de tener en cuenta la multimodalidad es especialmente relevante.

Si una de las funciones del lenguaje es la metalingüística, la capacidad de reflexionar sobre el lenguaje, debería considerarse también la posibilidad de desarrollar la función metamultimodalitat, es decir, la capacidad de reflexionar sobre los otros modos semióticos, especialmente cuando se trabaja con textos que son híbridos semióticos, como es el caso de los trabajos de investigación (Pujol Dahme, 2014).

\section{CONCLUSIONES E IMPLICACIONES DIDÁCTICAS}

En este artículo hemos visto algunos aspectos de las dimensiones lingüística, discursiva y la semiótica visual. De la dimensión lingüística se han revisado las características léxico-gramaticales como la diversidad léxica, la complejidad sintáctica y la densidad léxica. Estas son características de la escritura académica y, a su vez, se ha sugerido que pueden ser reflejo del desarrollo de las habilidades de escritura de los estudiantes. No obstante, llegar a adecuar estas características a las convenciones de la comunidad disciplinar es un desarrollo que requiere tiempo y sobre todo práctica.

De la dimensión discursiva se ha destacado la importancia de la argumentación para la comunidad científica y como proceso para planificar la escritura académica de los estudiantes. Se ha propuesto el modelo de Toulmin, que se utiliza con frecuencia en los niveles universitarios, tanto para el análisis como para la práctica argumentativa. Generalmente en las asignaturas de ciencias los estudiantes no desarrollan un aprendizaje profundo de la escritura; no obstante, esta sirve para entender mejor el contenido y asimismo ayuda a desarrollar la competencia argumentativa de las diferentes disciplinas. Desde las macro funciones de la Lingüística Sistémico Funcional se ha visto cómo se puede formar 'significado' con el modo visual y se ha constatado que no se puede asumir que el significado sea exclusivo del lenguaje verbal, escrito $u$ oral. Por el contrario, una visión centrada sólo en algunos géneros en los que predomina el texto verbal (novelas, artículos filosóficos, etc.) no permite ver la capacidad comunicativa de la combinación de otros modos semióticos, en este caso concreto, los textos científicos. Si se utiliza adecuadamente la variedad de modos semióticos, estos consiguen amplificar el significado; una gráfica, un mapa 0 una imagen contribuyen a dar un significado global. Este, sin embargo, no es la suma de los diferentes fragmentos significativos, sino que la totalidad es mucho más significativa. En una época en que los estudiantes están inmersos en modos semióticos visuales, tener en cuenta la metamultimodalidad, la reflexión sobre otros modos semióticos, 
permitiría un conocimiento más profundo para poder combinarlos de forma adecuada y así extraer todo su potencial.

Estas dimensiones solo son una parte de lo que implica llegar a utilizar y gestionar adecuadamente el lenguaje académico, en general, y el científico, en particular. Es un proceso que necesita práctica y además en él intervienen factores como la alfabetización académica, la madurez cognitiva, restricciones del tipo de género y factores sociales, ya que la el lenguaje académico escrito está determinado culturalmente. Por todos estos factores, el desarrollo de las habilidades de escritura supone un reto para los estudiantes. Para hacer frente a este desafío, se destaca el valor didáctico de la utilización de corpus en la enseñanza de la escritura académica. Un corpus permite el estudio de la 'lengua en uso', ya que generalmente refleja la variación lingüística en el discurso. Las opciones de todo el repertorio lingüístico se pueden explicar mejor y ser entendidos por los estudiantes, ya que están relacionados con el discurso auténtico. El uso de corpus podría ser un instrumento efectivo para los estudiantes, sobre todo, en niveles avanzados de la escritura académica, ya que cada comunidad discursiva utiliza características específicas del discurso. Por consiguiente, los ejemplos extraídos de un corpus, permiten ver cómo el repertorio lingüístico se utiliza de un modo retórico flexible, es decir, se adapta según el contexto comunicativo.

\section{REFERENCIAS BIBLIOGRÁFICAS}

Baratta, M. (2010). Nominalization development across an undergraduate academic. Journal of Pragmatics, 42(4), 1017-1036.

Bar-llan, L. y Berman, R. A. (2007). Developing register differentiation: The Latinate-Germanic divide in English. Linguistics, 45(1), 1-35.

Bazerman, C. (1988). Shaping written knowledge: The genre and activity of the experimental article in science. Madison, Wl: The University of Wisconsin Press.

Bazerman, C. (2005). Communication in the scientific community. En S. Restivo, Science, technology, and society: An encyclopedia (pp. 55-61). New York, NY: Oxford University Press.

Bereiter, C. y Scardamalia, M. (1987). The psychology of written composition. Hillsdale, NJ: Lawrence Erlbaum.

Berman, R. A. (2007). Developing linguistic knowledge and language use across adolescence. En E. Hoff y M. Schatz, Blackwell handbook of language development (pp. 347-367). Malden, MA: Blackwell Publishing.

Berman, R. A. y Verhoeven, L. (2002). Cross-linguistic perspectives on the development of text-production abilities: Speech and writing. Written Language and Literacy , 5(1), 1-43.

Biber, D. (1995). Dimensions of register variation: A cross-linguistic comparison. Cambridge: Cambridge University Press. 
Biber, D. (2006). University Language: A Corpus-based study of spoken and written registers. Amsterdam, Netherlands: John Benjamins.

Biber, D. y Gray, B. (2010). Challenging stereotypes about academic writing: Complexity, elaboration, explicitness. Journal of English for Academic Purposes, 9(1), 2-20.

Biber, D., Gray, B. y Poonpon, K. (2011). Should we use characteristics of conversation to measure grammatical complexity in L2 writing development? TESOL Quarterly, 45(1), 5-35.

Caamaño, A. (2010). Argumentar en ciencias. Un elemento esencial para la educación científica y ciudadana. Revista Alambique. Didáctica de las ciencias experimentales, 63, 5-10.

Carlino, P. (2005). Escribir, leer y aprender en la universidad. Una introducción a la alfabetización académica. Buenos Aires, Argentina: Fondo de cultura económica.

Carlino, P. (2013). Alfabetización académica diez años después. Revista mexicana de investigación educativa, 18(57), 355-381.

Chafe, W. y Danielewicz, J. (1987). Properties of spoken and written language. En R. Horowitz y S. J. Samuels (Eds.), Comprenhending oral and written language (pp. 83-113). San Diego, California: Academic Press.

Christie, F. y Derewianka, B. (2008). School discourse: Learning to write across the years of schooling. London, U.K.: Continuum.

Colombi, M. (2002). Academic language development in Latino Students' writing in Spanish. En M. Schleppegrell y M. Colombi, Developing Advanced Literacy in First and Second Languages. (pp. 67-86). Mahwah, NJ: Erlbaum.

Conrad, S. (1996). Investigating academic texts with corpus-based techniques: An example from biology. Linguistics and Education, 8, 299-326.

Driver, R., Newton, P. y Osborne, J. (2000). Establishing the norms of scientific argumentation in classrooms. Science Education, 84(3), 287-313.

Gardner, S. y Nesi, H. (2013). A classification of genre families in university student writing. Applied Linguistics , 34(1), 25-52.

Halliday, M. (1978). Language as social semiotic: The social interpretation of language and meaning. Baltimore: University Park Press.

Halliday, M. (1993). Some grammatical problems in scientific English. En M. Halliday y J. R. Martin (Eds.), Writing science: Literacy and discourse power (pp. 69-85). Pittsburgh, PA: University of Pittsburgh Press.

Halliday, M. A. (2004). The language of science. New York, NY: Continuum. Halliday, M. A. y Hasan, R. (1989). Language, context, and text: Aspects of language in a social-semiotic perspective (2nd ed.). Oxford: Oxford University Press.

Halliday, M. y Martin, J. R. (1993). Writing science: Literacy and discourse power. Pittsburg, PA: University of Pittsburgh Press.

Hyland, K. (2004). A convincing argument: Corpus analysis and academic persuasion. En U. Connor y T. A. Upton (Eds.), Discourse in the 
professions: Perspectives from corpus linguistics (pp. 87-112). Amsterdam: John Benjamins.

Ivanic, R. (1998). Writing and identity. The discoursal construction of identity in academic writing. Amsterdam, Netherlands: Benjamins.

Johansson, V. (2008). Lexical diversity and lexical density in speech and writing: A developmental perspective. Working Papers (Lund University), 53, 61-79.

Kress, G. y Van Leeuwen, T. (1996). Reading images: The grammar of visual design. London: Routledge.

Kucer, S. (2005). Dimensions of Literacy: A conceptual base for teaching reading and writing in school settings. Mahwah, $\mathrm{NJ}$ : Lawrence Erlbaum Associates.

Lemke, J. (1989). Multimedia demands of the scientific curriculum. Linguistics and Education, 10(3), 247-272.

Lemke, J. (1998). Multiplying Meaning: Visual and Verbal Semiotics in Scientific Text. En J.R. Martin \& R. Veel (Eds.), Reading Science. London. Routledge, pp. 87-113.

Lemke, J. (1990). Talking science: Language, learning, and values. Norwood, NJ: Ablex.

Lemke, J. (2002). Making meaning across textscales: A critical statement on reading comprehension. RAND Reading Study Group.

Malvern, D., Richards, B., Chipere, N. y Durán, P. (2004). Lexical diversity and language development: Quantification and assessment. New York, NY: Palgrave Macmillan.

Martin, J. R. (1984). Language, register and genre. En F. Christie, Children writing (pp. 21-29). Geelong, Australia: Deakin University Press.

Matthiessen, C. (2009). Léxico-gramática y colocación léxica: Un estudio sistémico-funcional. Signos, 42(71), 333-383.

Norris, S. y Phillips, L. (2009). Scientific literacy. En D. Olson y N. Torrance (Eds.), The Cambridge handbook of literacy (pp. 271-285). Cambridge, UK: Cambridge University Press.

Prior, P. (2006). A sociocultural theory of writing. En C. McArthur, S. Graham y J. Fitzgerald, Handbook of writing research (pp. 54-66). New York, NY: Guilford Press.

Pujol Dahme, A. (2014). Llegim imatges: La gramàtica visual als treballs de recerca de batxillerat. En L. Tolchinsky i M. Fons (Ed.), Articles. Didàctica de la llengua i la literatura. La lingüística de corpus a l'aula (pp. 45-53). Barcelona: Ed. Graó.

Pujol Dahme, A. y Selfa, M. (2015) The transition from university to publication: Register and interactional metadiscourse features in immunology research written in Catalan and English. Ibérica. Revista de la Asociación Europea de Lenguas para Fines Específicos 30(1), 155-182.

Rodríguez Bello, L. (2004). El modelo argumentativo de Toulmin en la escritura de artículos de investigación educativa. Revista Digital Universitaria 
http://www.revista.unam.mx/vol.5/numl/art2/art2.htm, [Consulta: 05 de mayo de 2012].

Russell, D. R. y Cortes, V. (2012). Academic and scientific texts: the same or different communities? En M. Castelló y C. Donahue (Eds.), University writing. Selves and texts in academic societies (pp. 3-18). Bingley, UK: Emerald.

Sampson, V. y Clark, D. B. (2008). Assessment of the ways students generate arguments in science education: Current perspectives and recommendations for future directions. Science Education, 92(3), 447-472.

Sandoval, W. A. y Reiser, B. J. (2004). Explanation driven inquiry: Integrating conceptual and epistemic scaffolds for scientific inquiry. Science Education, 88(3), 345-372.

Schleppegrell, M. J. (2001). The linguistic features of the language of schooling. Linguistics and Education, 12(4), 431-459.

Schleppegrell, M. J. y Colombi, M. C. (2002). Theory and practice in the development of advanced literacy. En M. J. Schleppegrell y M. C. Colombi (Eds.), Developing advanced literacy in first and second languages: meaning with power (pp. 1-19). Mahwah, NJ: Lawrence Erlbaum.

Snow, C. E. y Uccelli, P. (2009). The challenge of academic language. En D. R. Olson y N. Torrance (Eds.), The Cambridge handbook of literacy (pp. 112-133). Cambridge: Cambridge University Press.

Snow, C. y Biancarosa, G. (2003). Adolescent literacy and the achievement gap: What do we know and where do we go from here? New York, NY: Carnegie Corporation.

Swales, J. M. (2004). Research genres: Explorations and applications. New York, NY: Cambridge University Press.

Tolchinsky, L. y Simó, R. (2001). Escribir y leer a través del currículum. Cuadernos de educación, (pp.159-165). Barcelona: ICE/Horsori.

Tolchinsky, L., Castrechini, A., Cañedo, M., Estrada, F., Gracia, M. C., Navarro, M., Pujol Dahme, A. (2013). La escritura académica a través de las disciplinas. Barcelona: Octaedro.

Uccelli, P., Dobbs, C. y Scott, J. (2013). Mástering academic language: Organization and stance in the persuasive writing of high school students. Written Communication, 30(1), 36-62.

Veel, R. (1997). Learning how to mean scientifically speaking: apprenticeship into scientific discourse in the secondary school. En F. Christie y J. R. Martin, Genre and Institutions: Social Processes in the Workplace and School (pp. 161-195). London, U.K.: Cassell.

Ventola, E. (1996). Packing and unpacking of information in academic. En E. Ventola y A. Mauranen, Academic Writing. Intercultural and textual issues (pp. 153-194). Philadelphia, PA: Benjamins.

White, B. y Frederiksen, J. (1998). Inquiry, modeling, and metacognition: Making science accessible to all students. Cognition and Instruction, 16, 3-118. 
Woerfel, T. y Yilmaz, S. (2011). Lexical Development of German-Turkish bilinguals:of German-Turkish bilinguals: A comparative study in written discourse. En C. Cummins (Ed.), Proceedings of the 6th Cambridge Postgraduate Conference in Language Research (pp. 240-251). Cambridge: Cambridge Institute of Language Research.

\section{Sobre la autora:}

Ana Pujol Dahme

Ana Pujol Dahme es profesora asociada del Departamento de Didáctica de la Lengua y la Literatura de la Facultad de Educación de la Universidad de Barcelona. Es una investigadora en formación del programa de doctorado en Lingüística Teórica, Computacional y Aplicada (UB). También es miembro del Grup de Recerca del Repertori Lingüístic (GRERLI) de la Universidad de Barcelona. Sus intereses incluyen la escritura académica, la semiótica visual y la lingüística basada en corpus.

\section{Para citar este artículo}

Pujol Dahme, A. (2015). La alfabetización académica desde una perspectiva multidimensiona. Revista Fuentes, 17, Diciembre, pp. 6787. [Fecha de consulta: $\mathrm{dd} / \mathrm{mm} / \mathrm{aa}$ ]. doi: doi: http://dx.doi.org/10.12795/revistafuentes.2015.i17.03. 